\title{
Physico-chemical characteristics of Longissimus lumborum muscle in goats subjected to halal slaughter and anesthesia (halothane) pre-slaughter
}

\begin{abstract}
This study assessed the effect of halal slaughter and anesthesia pre-slaughter followed by bleeding on meat quality characteristics of goats. Eleven male Boer cross goats were divided into two groups and subjected to either halal slaughter (HS) or anesthesia with halothane and propofol pre-slaughter (AS). At pre-rigor, HS had significantly lower (P and glycogen than AS. However, no significant difference was observed in the $\mathrm{pH}$ and glycogen content between the treatments on 1, 3 and 7 days post mortem. The drip loss of HS was significantly lower (P\&Wl.05) than that of AS at all aging periods. Treatment had no effect on sarcomere length, myofibrillar fragmentation index and shear force values, loss of thiol groups and degradation of major myofibrillar proteins. It can be concluded that HS did not have deleterious effect on meat quality traits of goat when compared to AS.
\end{abstract}

Keyword: Goat; Halal slaughter; Meat quality; Minimal anesthesia 